The agenda was made up of some seventy technical papers in addition to the reports of the subcommittees on Service psychology and defence food research and the group on agricultural aspects of defence science. The papers were circulated in advance, and at the Conference they were introduced briefly by named delegates and then discussed by the whole meeting. The papers fell largely in the broad fields covered by the above groups and in the fields of career development, telecommunications, tropical warfare, weapons testing, naval research cold-weather operations, effects of high-level noise and the effects of nuclear weapons. However, in addition, there were many individual papers outside these groups.

The subject of tropical warfare was one of special interest to several of the countries represented, in that their territories embraced large areas of tropical terrain. Numerous papers were discussed, ranging from the effect of the environment on the soldier and his equipment, the need for more efficient vehicles and aircraft for use in tropical areas, mental stress under tropical conditions, navigation and communication in the jungle, clearance of vegetation, corrosion in the tropics, etc.

The weapons-testing theme covered the biggest group of papers discussed at the Conference. The subject was interpreted in its broadest sense, aeroplanes, for example, being treated as weapons. This theme was of great interest to Australia, where two of the major defence science activities, namely, the Woomera Missile Testing Range and the Maralinga Atomic Testing Range, are both concerned very largely with the testing of weapons.

In particular, the Woomera project was discussed in some detail. Numerous papers dealing with the equipment of the Range, various aspects of the trials work and the processing of the results were considered. The discussions were supplemented by a comprehensive visit to Woomera, when the facilities were examined in detail and missile trials were witnessed.

While at Woomera, delegates inspected the missiletracking station which had been established as part of the International Geophysical Year programme.

The visit to Woomera was of particular interest to delegates. The basic conception of this project arose largely from the early discussions by the Commonwealth Advisory Committee on Defence Science, and it was a matter of considerable satisfaction to dele. gates to see the very tangible result that had been produced. Sir John Carroll, leader of the British delegation, in a public address, referred to Woomera as the outstanding example of defence science co-operation in the Free World.

Because of the importance attached to seeing at first hand something of the scientific work proceeding in Australia, several visits were arranged to scientific and appropriate Service establishments. Included in these were the Aeronautical Research Laboratories, the Defence Standards Laboratories, the Weapons Research Establishments at Salisbury and the testing range at Woomera, the Maralinga Atomic Testing Establishments and the Edinburgh and Woomera Royal Air Force Establishments.

Quite apart from these visits, it was felt that it would be of interest for delegates to be given opportunities of seeing something of the country and of work proceeding in other technical fields. For this purpose, a two-day visit was arranged to the Snowy Mountains Scheme in the south-eastern corner of Australia. This is the largest engineering undertaking in Australia, and can compare in magnitude with the largest undertakings in other parts of the world. Although primarily concerned with the conservation of water by means of river diversion and large-scale storage, it is also a major generator of electric power, having a potential capacity of some $2,000,000 \mathrm{~kW}$.

Numerous opportunities were included in the programme to enable delegates to meet Australians in many walks of life. Delegates were honoured by being entertained to lunch by the Government of the Commonwealth and by being received by the Governors of Victoria and South Australia and by the Lord Mayor of Melbourne. In addition to such official functions, several pleasant informal functions including visits to places of interest and private entertainments were arranged-visits to outback sheep stations and to the Andamooka opal fields.

At the close of the Conference, delegates were unanimous that it was one of the most successful held to date. The tangible results are, of course, a series of recommendations arising from three weeks of intensive discussions. These recommendations are for the guidance of member countries; they will be submitted to the respective governments for ratification, when they will become a valuable guide to defence science policies. The other result of the Conference is the intangible one arising from the friendships established between delegates and the mutual appreciation of the special problems faced by member countries.

\section{Lestife Martin}

\title{
AUTOMATIC REDUCTION OF GEOPHYSICAL DATA
}

$\mathrm{O}$ VER the past two decades there has been a rapid increase in the use of automatic photographic and electronic techniques for recording experimental results. As a result, the number of measurements made need no longer be restricted by fatigue in the experimenter, and sets of results containing millions of measurements are not uncommon. The rate at which results can be accumulated is now so great that the individuals responsible for setting up the equipment can no longer assimilate the information at the rate at which it is produced. This problem can be overcome to some extent by arranging the programme of analysis in such a way that the reduction of data can be dealt with by semi-skilled staff.

Nowadays even this solution is often inadequate. Not only is it difficult to find suitable staff, but also many of the investigations require the analysis of data on a scale so great as to be beyond the capabilities of a team of individuals. The problem is particularly acute in the field of geophysical research, where very large quantities of results are frequently produced in the course of field work of many kinds. 
The automatic reduction of geophysical data was the subject of a Geophysical Discussion organized by the Royal Astronomical Society and held on January 23 under the chairmanship of Sir Edward Bullard. Although the discussion was not primarily concerned with the results of the International Geophysical Year, it was appropriate that it should have been arranged at a time when many individuals must be giving consideration to the study of the unprecedented wealth of new information obtained during the preceding eighteen months.

Sir Edward opened the discussion by pointing out that a single measurement made in each degree square of the Earth's surface would amount to a total of nearly 70,000 readings, and that facilities for handling large numbers of data are almost essential in many types of geophysical work. Even in mobile experiments it was most desirable to arrange, if at all possible, for the results to be recorded directly in digital form. This saved a great deal of time later, since it avoided the need for manual punching on tape or cards, and it frequently made it possible to record data at a rate which would not be possible by other methods.

Sir Edward described his recent voyage in Discovery $I I$, in the course of which 90,000 magnetic readings were made near the Azores. The results were recorded directly on punched tape together with other essential information which would be needed during the reduction stage. These included the date, time, records of clock adjustments and range changes and also the ship's position and heading. The programme for the computer was written in such a way that the whole reduction process could be dealt with in one stage. As the principal objective of the work was the study of small irregularities in the Earth's field, it was arranged that the regular component of the field should be removed as part of the computer programme.

Mr. R. H. Merson (Royal Aircraft Establishment) described some investigations of the orbits of artificial Earth satellites. The original aim of the work was to predict the times of future transits in order to enable more accurate positional data to be recorded: Later, it was found possible to gain valuable geodetic information from the results. The approximate size and shape of the orbit are first determined on a desk calculator. This orbit is then adjusted by successive approximations, using a digital computer, to give a least-squares fit with all the available observational information.

The accuracy of the observations is such that it has been possible to derive new values for the coefficients $J$ and $D$ in Jeffreys's expression for the Earth's gravitational potential and to make deductions concerning atmospheric drag on satellites.

The prediction of future transits of Earth satellites requires the rapid processing of incoming data if the results are to be produced in time to be of use. The conditions under which weather forecasts are produced are similar and provide an obvious reason for the use of a digital computer. Mr. G. A. Corby (Meteorological Office) discussed preliminary tests on the preparation of numerical forecasts with the aid of a computer. Barometric pressure and wind-speed measurements are received on tape in a variety of codes at a rate of about 100,000 a day and, to save time, the incoming tapes are decoded in the computer before being used for further processing. The object is to construct a contour map, showing the height of a constant-pressure surface over Europe and the North Atlantic, which accurately represents the observational data. In the least-squares fitting process, it is necessary to minimize the differences between the observed and calculated values of both pressure and wind speed. An additional requirement is that in the determination of the contours for any area, maximum weight must be given to the data from the nearest weather stations. The speed and accuracy with which the forecasts can be produced by automatic methods are satisfactory even though the techniques are still in the development stage.

Mr. M. H. P. Bott (University of Durham) discussed the use of a digital computer for determining the extent of geological formations from surface gravity anomalies. The anomaly due to a model consisting of a series of vertical rectangular prisms of arbitrary depth has been calculated. In the study of a particular formation the procedure adopted is to define a model which leads to the same calculated anomaly as that observed in the area under investigation. Providing the density of the formation is known, the form of the lower surface can be determined uniquely by this method. The technique has been successfully used on the Triassic rocks in Dumfries.

Dr. D. C. Martin (Royal Society) outlined briefly the arrangements which had been made for the collection of data during the International Geophysical Year. Full sets of data would be held in World Data Centres and selected data would be published in Annals of the International Geophysical Year. Although no plans had been made in advance for the automatic processing of the whole of these data, it seemed likely that much of the information would eventually be dealt with by machine methods.

Mr. M. J. Tucker (National Institute of Oceanography) discussed various techniques used in the spectral analysis of geophysical time-series with particular reference to the study of sea waves. Although the analysis can be carried out by deriving the Fourier components of the wave motion, it is often more satisfactory to express the results in terms of the power spectrum or the autocorrelation function.

Proposals for the application of a digital computer to the forecasting of the future behaviour of the ionosphere were outlined by Mr. C. M. Minnis (Radio Research Station). Present intentions are to represent the characteristics of the $F$-layer in terms of a family of linear functions of solar activity based on all the past observational data. This can be achieved to a satisfactory degree of accuracy if an ionospheric index is used to represent solar activity. The intercepts and slopes which define the linear relations would be stored in a digital computer and those needed to make a forecast for a specified location, season, time of day and level of solar activity would be selected and used in conjunction with other data, in the later stages of the calculations. It is hoped that by this means it will be possible to make forecasts of a more practically useful type than those made using standard manual methods.

In many types of geophysical investigation the use of an automatic digital computer is almost essential if accurate results are to be produced quickly, but it must not be assumed that this is always so. Three speakers referred to work which was being carried out successfully and economically by other methods.

Mr. A. Dennison (British Petroleum Company) described the echo method for the exploration of underground geological formations and the difficulties 
associated with the usual 'single spot' method of recording the results. Variable-density and variablearea recording had also been tried and the resulting records, when suitably displayed, could give a striking visual picture of the density variations in the underlying structures without any of the computations normally associated with echo soundings.

The analysis of Earth tide records obtained using Milne-Shaw pendulums was explained by Mr. G. W. Lennon (Liverpool Observatory and Tidal Institute), who enumerated reasons why a digital computer would not be appropriate in this type of work. For example, the removal of spurious diurnal and semidiurnal oscillations would be difficult to programme for a computer, quite apart from the impossibility of producing long records without discontinuities.

Finally, Mr. H. M. Iyer (National Institute of Oceanography) referred to the lack of interest in the study of microseisms. If properly analysed they could yield useful information, and Mr. Iyer described experimental equipment which had recently been developed for the automatic recording of several statistical characteristics of microseisms.

C. M. Minnis

\section{CHEMISTRY OF CONDENSED TANNINS}

$\mathrm{T}$ HE Plant Phenolics Group held a meeting on "The Chemistry of Condensed Tannins" at Queen Elizabeth College, University of London, on January 6 under the chairmanship of Prof. R. D. Haworth (University of Sheffield). More than sixty members and guests were present.

In the opening paper on "Acid-catalysed Polymerization of Phenolic Compounds", Dr. B. R. Brown (Oxford) briefly summarized past work on this subject and indicated a possible reaction mechanism. $p$-Methoxybenzyl alcohols condense with phenols preferentially in a position para to a hydroxyl group to yield diphenylmethanes. A suitably substituted flavan, for example 4'-methoxyflavan, undergoes this condensation via ring-fission. Condensation of 4'-methoxyflavan with 7-hydroxyflavan yields a compound containing two flavan units the structure of which is based upon the known para-orientation of these reactions.

The importance of flavan-3:4-diols in tannin chemistry has led to an investigation of flavan-4-ol, which condenses with phenols, and with 7-hydroxyflavan yields a flavan 'dimer' isolated as the crystalline acetate.

These condensations, like the acid-polymerization of catechin, require high acid concentrations and it is unlikely that they are involved in the conversion of catechins into tannins in plants. This work aims at establishing the structural details of this condensation and at producing 'polymers' of known structure which can be used for the trial of new degradative methods, which may also be valuable for the degradation of condensed tannins.

Dr. D. E. Hathway (British Leather Manufacturers' Research Association), in a paper entitled "Oxidative Polymerization Mechanisms for Catechins", first briefly reviewed the chemistry of the oxidation of $o$ - and $p$-dihydroxy- and vic-trihydroxyphenols, which he illustrated by reference to the synthesis of the mould metabolite atromentin, to the formation of purpurogallin and the synthetic melanins, and to the transformation of gallates into ellagates. Possible mechanisms for the oxidation of coniferyl alcohol by laccase, and their relevance to lignin and lignan formation were also discussed.

Studies on the oxidation of $(+)$-catechin in aqueous and non-aqueous media and on the autoxidation of flavans related to catechin confirm that autoxidation of catechin to a polymer (catechin-tannin) proceeds via the corresponding $o$-quinone followed by intermolecular C-C linkage. The evidence suggests that the most likely linkage is between the 8-carbon of one molecule and the 6'-carbon of the second. The isolation, albeit in small yield, of $m$-hemipinic acid by oxidative degradation of the methylated reduced polymer substantiates this supposition. Further confirmation for the presence of head-to-tail units in the polymer was obtained from a study of the autoxidation and the enzymic oxidation of mixed substrates which resemble the head and tail of $(+)$-catechin. Enzymic oxidation by mushroom, potato and tobacco polyphenoloxidases proceeds at a faster rate and lower temperature than the autoxidation of catechin, and gives a product precisely similar to the autoxidation polymer.

The heartwood of Acacia catechu and the leaves of Uncaria gambir contain considerable quantities of (-)-epicatechin and (+)-catechin, respectively. The isolation, therefore, of purified tanning fractions in good yield from these sources, with properties similar to the autoxidation and enzymic oxidation polymers of catechin, strongly suggests that these tannins are formed from epicatechin and catechin, respectively, by the above mechanism.

Studies on the formation of oak-bark tannin indicate that $(+)$-catechin, $(+)$-gallocatechin and leucodelphinidin, which are formed in the leaves, are translocated via the sieve-tube system of the phloem to the cambium, where preferential enzymic oxidation of the pyrogallol phenols occurs. The resulting tail-to-tail polymer is stored in the bark. The remaining tannin action of the bark can be attributed to the occurrence of complex leucoanthocyanins.

In a paper on "Oxidative Condensation in Tea Fermentation", Dr. E. A. H. Roberts (Indian Tea Association) said that, according to Hathway and Seakins, oxidation of $(+)$-catechin is followed by a condensation between the catechol-o-quinone group of one molecule and the phloroglucinol ring of another. If phloroglucinol is added to an enzymecatechol system, different intermediate- and endproducts are formed, indicating a condensation of o-benzoquinone with highly active phloroglucinol. Oxidation of pyrogallol to purpurogallin, however, is not affected by the presence of phloroglucinol, which illustrates the very high reactivity associated with the hydroxy-o-benzoquinone system. Enzymic oxidations of (-)-epigallocatechin and its gallate are similarly unaffected by the addition of phloroglucinol, but the addition of pyrogallol to these systems yields derivatives of purpurogallin (flavanotropolones). These observations suggest that, following oxidation 